\title{
Essais
}

ESSAIS

Revue interdisciplinaire d'Humanités

$7 \mid 2015$

Normes communiquées, normes communicantes

\section{Normes communiquées, normes communicantes}

Avant-propos

Laetitia Biscarrat et Clément Dussarps

\section{CpenEdition}

Journals

Édition électronique

URL : http://journals.openedition.org/essais/6110

DOI : $10.4000 /$ essais. 6110

ISSN : 2276-0970

Éditeur

École doctorale Montaigne Humanités

Édition imprimée

Date de publication : 1 décembre 2015

Pagination : 8-13

ISBN : 978-2-9544269-6-9

ISSN : $2417-4211$

Référence électronique

Laetitia Biscarrat et Clément Dussarps, "Normes communiquées, normes communicantes », Essais

[En ligne], 7| 2015, mis en ligne le 26 février 2021, consulté le 27 février 2021. URL : http://

journals.openedition.org/essais/6110; DOI : https://doi.org/10.4000/essais.6110 


\section{Avant-propos}

\section{Laetitia Biscarrat, Clément Dussarps}

Comment questionner les normes dans une perspective communicationnelle? Tel fut l'enjeu de la journée d'études doctorales " Normes communiquées, normes communicantes : assignations, déviances et négociations " qui s'est tenue le 6 juin 2013 à l'Espace Histoire-Image de la Médiathèque de Pessac ${ }^{1}$. Avant de lire les réponses formulées par les contributrices de ce dossier, quelques rappels s'imposent. Comme l'a montré Foucault ${ }^{2}$, nous vivons dans une société de normes, qui sont autant de rapports de pouvoir structurant à la fois l'individu et le collectif. La norme est une relation de pouvoir à la fois productrice et stabilisatrice. Elle façonne des comportements et génère des attentes d'une plus ou moins grande conformité selon une échelle de valeur normative. Pour autant, les normes ne sont pas strictement extérieures aux individus. Le sujet chez Foucault est produit par des techniques de pouvoir et des dispositifs de contrôle. C'est un individu historiquement situé dont les modalités d'existence sont fondées sur l'expérience sociale. Cet individu est intimement aux prises avec les normes. "L'homme dont on nous parle et qu'on invite à libérer est déjà en lui-même l'effet d'un assujettissement bien plus profond que lui. Une “âme” l'habite et le porte à l'existence, qui est ellemême une pièce dans la maîtrise que le pouvoir exerce sur le corps. » ${ }^{3}$

1 Cette journée fut organisée à l'initiative de l'association des doctorant.e.s de l'équipe d'accueil MICA, Université Bordeaux Montaigne. Nous remercions chaleureusement les partenaires et soutiens institutionnels et financiers parmi lesquels l'Université Bordeaux Montaigne, le MICA, la Mairie de Pessac, la Banque Populaire Centre Atlantique et l'Atelier Genre de l'Université Bordeaux Montaigne. Nous remerciements se tournent également vers les modérateurs et intervenants qui ont assuré la réussite de cette journée : Maxime Cervulle, Nelly Quemener et Geneviève Sellier pour les plénières, mais aussi Manuel Billi, Charlotte Blanc, Pascale Caemerbeke, Delphine Chedaleux, Karine Espineira, Fanny Gonzalez, Julie Laffont, Sarah Lécossais, AnneCécile Lenoël, Anne Lubnau, Myriam Hernández Orellana, Thomas Pillard, Juliette Raynaud, Jules Sandeau, Catherine Souladié.

2 Michel Foucault, Surveiller et punir, Paris, Gallimard, 1975.

3 Ibid., p. 38. 
La relecture des travaux de Foucault par Judith Butler apporte un éclairage sur les liens entre normes, société et individu. Pour la philosophe, «le sujet est l'effet rétroactif du pouvoir $»^{4}$. Les normes préexistent à un individu qui leur est perméable. Ainsi, la norme désigne la relation entrel'individu et le pouvoir. «Aucun individu ne devient sujet sans être d'abord assujetti et sans subir une sujétion. " ${ }^{5}$ La subordination au pouvoir produit un sujet intelligible socialement. On est humain parce qu'on a d'abord été assujetti. Paradoxalement, l'assujettissement au pouvoir est donc un processus de subjectivation. Le sujet est soumis au pouvoir, mais il est également produit par cette sujétion. Sa capacité d'agir n'est rendue possible que par cet assujettissement inaugural. "L'“assujettissement" désigne à la fois le processus par lequel on devient subordonné à un pouvoir et le processus par lequel on devient un sujet ", écrit Butler.

La notion d'" effet " du pouvoir évoquée par Butler porte une seconde proposition. En plaçant l'humain en position adjective aux rapports de pouvoir, elle remet en cause la vision cartésienne du sujet moderne. "Le sujet souverain est ainsi une fiction théorique que l'on peut déconstruire. ${ }^{7}$ Loin d'un cogito " qui s'auto-interpelle $»^{8}$, l'individu-effet-du-pouvoir incarne, reproduit et parfois négocie ou conteste les normes hégémoniques. Comme l'indique l'étymologie latine effectus, un effet est une exécution, une réalisation, un résultat. Et, parfois, ce que le langage courant désigne comme un " effet secondaire " surprend, autrement dit déroge aux attentes supposées, normées. Les normes autorisent dès lors une hypothétique "marge de manœuvre ». À propos des normes de genre, Butler explique que le sujet les cite de manière répétée, l'identité désignant alors ce processus de sédimentation'. La norme se construit sur un mode itératif. Elle existe au travers de sa répétition. " La norme procède de la fréquence de son apparition : par là est révélé son caractère productif. $»^{10}$ Une norme qui ne serait pas actualisée cesserait donc d'en être une. La réactualisation continue des normes est donc porteuse d'un potentiel de négociation, de subversion voire de contestation. Citer le genre « c'est aussi le faire à sa manière, qui change imperceptiblement d'une fois à l'autre, comme un pianiste ne rejoue jamais de la même façon une sonate de Beethoven ${ }^{11}$.

4 Judith Butler, La vie psychique du pouvoir, L'assujettissement en théories, traduit de l'anglais par Brice Matthieussent, Paris, Léo Scheer, 2002, p. 28.

5 Ibid., p. 34.

6 Ibid., p. 23.

7 Fabienne Brugère et Guillaume Le Blanc, Judith Butler. Trouble dans le sujet, trouble dans les normes, Paris, PUF, 2009, p. 18.

8 Elsa Dorlin, Sexe, genre et sexualités, Paris, PUF, 2008, p. 77.

9 Judith Butler, Trouble dans le genre. Pour un féminisme de la subversion, traduit de l'anglais par Cynthia Kraus, Paris, La Découverte, 2005.

10 Fabienne Brugère et Guillaume Le Blanc, op. cit., p. 13.

11 Marie-Joseph Bertini, Ni d'Eve ni d'Adam. Défaire la différence des sexes, Paris, Max Milo, 2007, p. 79. 
Nous pouvons dire que la norme est productrice tout autant qu'elle est produite par les usages qui en sont faits. "Si la norme n'est pas extérieure à son champ d'application, ce n'est donc pas seulement parce qu'elle le produit, mais parce qu'elle s'y produit elle-même en le produisant ${ }^{12}$, explique Pierre Macherey. Parmi ces champs d'application, celui de la culture - pris au sens large, de la culture d'élite aux " médiacultures " ${ }^{13}$ - constitue un poste d'observation privilégié du travail de réactivation permanente des normes. En effet, les productions culturelles portent la trace des usages des normes. Ces usages opèrent au niveau de la production tout autant que de la réception. Dès lors, étudier les productions culturelles du point de vue des normes, c'est adopter une démarche pragmatique selon laquelle le contexte est constitutif de l'énoncé.

Un détour par l'appropriation que fait Stuart Hall de la notion gramscienne d'hégémonie éclaire cette approche des productions culturelles comme traces d'usage des normes. L'hégémonie est " une combinaison de puissance et de consentement ${ }^{14}$ qui opère sur le terrain des superstructures mais aussi des structures. Elle n'est pas figée et permanente mais en équilibre instable. Les représentations médiatiques témoignent pour Hall du travail idéologique qui est à l'œuvre pour l'imposition, le maintien et la négociation d'un ordre hégémonique. Les médias "servent à accomplir en permanence le travail idéologique essentiel consistant à "classer le monde" dans les discours des idéologies dominantes. " ${ }^{15}$ Les représentations sont donc normées, au sens où elles entrent dans un répertoire idéologique restreint qui favorise leur intelligibilité. Elles sont également normatives, dans la mesure où ces technologies de pouvoir re-produisent des rapports de domination qu'elles contribuent à naturaliser et rendre légitimes. L'effet idéologique de la culture et des médias évoqué par Hall opère dans la répétition plus ou moins à l'identique des normes, contribuant ainsi au consensus provisoire sur leur légitimité. Les productions culturelles sont dès lors une voie d'accès à la compréhension des normes qui sous-tendent la communication. Étudier les représentations donne à voir le travail des normes dans ces espaces de " média[tisa] tion " ${ }^{16}$ à la fois porteurs de normes et structurés par des normes.

12 Pierre Macherey, De Canguilhem à Foucault, la force des normes, Paris, La Fabrique, 2009, p. 90.

13 Éric Macé et Éric Maigret, Penser les médiacultures. Nouvelles pratiques et nouvelles approches de la représentation du monde, Paris, Armand Colin/INA, 2005.

14 Stuart Hall, "La culture, les médias et l'“effet idéologique" ", in Hervé Glevarec, Éric Macé et Éric Maigret (coord.), Cultural Studies. Anthologie, Paris, Armand Colin/INA, 2008, p. 43.

15 Ibid., p. 59.

16 Karine Espineira, "Un exemple de glissement du lexique médiatique : le sujet trans dans les productions audiovisuelles ", Essais, $\mathrm{n}^{\circ} 7$, Laetitia Biscarrat et Clément Dussarps (éd.), « Normes communiquées, normes communicantes ", 2015, p. 56. 
Les contributrices de ce dossier ont porté leur attention tout autant sur les productions culturelles des élites cultivées que sur la culture populaire des " médias de masse ". " "Prendre la fiction au sérieux" et la considérer comme un outil d'investigation du réel $\aleph^{17}$, telle est l'ambition des travaux de Taline Karamanoukian et Sarah Lécossais. Les auteures analysent le travail des normes de genre dans des fictions télévisuelles destinées au grand public. Leur propos n'est pas d'estampiller les représentations d'un label progressiste ou conservateur, mais d'étudier le travail de configuration des normes du féminin et de la maternité. Taline Karamanoukian nous invite à un voyage dans l'histoire de la télévision d'état française qui diffusa en 1963 son premier feuilleton télévisé sentimental, Janique Aimée, sur la Radiodiffusion-Télévision Française (RTF). Le feuilleton discute les normes du féminin dans une période d'émancipation pour les jeunes femmes, dont les horizons s'élargissent au-delà du foyer grâce à l'accès croissant aux études et au marché du travail. S'inscrivant dans une démarche pragmatique à l'intersection entre étude des normes et approche communicationnelle, l'auteure convoque les discours de la presse et de l'institution télévisuelle pour analyser les ambivalences du scénario et de la monstration de l'héroïne à partir d'une double approche sémanticosyntaxique et sémio-narratologique.

C'est également une étude des enjeux idéologiques des fictions télévisuelles que nous propose Sarah Lécossais dans une contribution consacrée aux configurations normatives de la maternité dans des séries françaises diffusées sur les chaînes hertziennes entre 1992 et 2012. L'auteure envisage la maternité comme un " métier ${ }^{18}$ structuré par un ensemble de normes dont l'effectivité repose sur un mécanisme de "consentement implicite à la domination ${ }^{19}$. Circonscrite à deux phénomènes emprunts de normativités, la culpabilisation des mères et le traitement de la grossesse, l'étude identifie dans un premier temps un idéal-type de la maternité dans les fictions. Il est structuré par quatre normes, qui sont autant d'impératifs : la communication, la responsabilité, l'amour et la réflexivité. Dans un deuxième temps, l'auteure confronte cette maternité idéale à des représentations contre-hégémoniques des mères dans la série Vous les femmes (M6, 2007-2010). Basée sur une succession de saynètes comiques, la série donne à voir tout autant qu'elle transgresse les normes de la bonne maternité, produisant ainsi un discours sur les normes qui est rendu possible par la dimension carnavalesque de cette parenthèse humoristique.

17 Marlène Coulomb-Gully, "Les femmes politiques au miroir des fictions télévisuelles. Commander in Chief et L'État de Grâce : une comparaison France/États-Unis ", Modern \& Contemporary France, vol. 20, n ${ }^{\circ}$ 1, 2012, p. 38.

18 Séverine Gojard, Le métier de mère, Paris, La Dispute, 2010.

19 Sarah Lécossais, "La maternité dans les séries familiales françaises : entre consensus et résistances ", Essais, n 7 , Laetitia Biscarrat et Clément Dussarps (éd.), « Normes communiquées, normes communicantes ", 2015, p. 31. 
La troisième contribution de ce dossier thématique ambitionne de penser les normes au-delà de la question du genre télévisuel. Karine Espineira examine la médiatisation des transidentités du point de vue de l'évolution du lexique médiatique. Elle s'appuie sur une discussion entre un travail d'observation participante du terrain transidentitaire français et une étude d'un corpus de plus de huit cents documents audiovisuels des années soixante-dix à nos jours. Cette confrontation souligne l'écart entre les normes identitaires hégémoniques du terrain et celles des représentations télévisuelles. Dans une perspective diachronique, l'auteure identifie les glissements du lexique médiatique qui font passer successivement la monstration des trans du fait divers au fait de société puis à la notion d'égalité des droits. Elle souligne ainsi le fonctionnement hégémonique, et dès lors évolutif, des représentations télévisuelles dont le genre (gender) apparaît comme un principe organisateur.

Les conflits de définition pour l'imposition d'une norme hégémonique qui fait sens commun ne sont pas le propre du média télévisuel. Charlotte Blanc nous fait part des résultats d'une étude exploratoire portant sur les normes communicationnelles et symboliques qui sous-tendent les réseaux traditionnalistes catholiques sur internet. Son corpus est circonscrit à la blogosphère auto-désignée de "réinformation " durant les débats sur le Mariage pour Tous (2012-2013), qu'elle étudie dans une approche systémique, à la fois analytique et interactionniste. Après avoir identifié la morphologie du réseau traditionnaliste, l'auteure étudie son intentionnalité communicationnelle à l'aune de la mobilisation symbolique et du militantisme discursif mis en œuvre dans les blogs. Leur homogénéité révèle la dimension normée tout autant que la visée normative de ces dispositifs communicationnels à la croisée des sphères spirituelle et politique.

C'est un autre discours minoritaire, soutenu par une bien moindre visibilité médiatique, qu'étudie Catherine Souladié. Quittant le champ de la religion pour celui des pratiques artistiques, l'auteure nous propose d'interroger le lien entre normes et corporéité au travers des pratiques de modifications corporelles de trois artistes emblématiques de ce champ de l'art contemporain : Albercht Becker, Ron Athey et Lukas Zpira. Leurs corps tatoués, percés, scarifiés, implantés et lacérés interrogent les normes du beau, de l'abject, de l'immoral. Ils mettent en exergue la productivité des normes par le biais de pratiques corporelles de subversion et de re-signification. Leur radicalité déconstruit le mythe d'une naturalité du corps pour en souligner la dimension politique et normée. Ces "stylisations de la chair ${ }^{20}$ sont pour l'auteure le symptôme d'un corps-médium, un corps communicant qui, au travers d'un questionnement des normes, donne à voir “ les bouleversements d'une époque et d'une société donnée » ${ }^{21}$.

20 Judith Butler, Trouble dans le genre, op. cit., p. 263.

21 Catherine Souladié, «Modifications corporelles dans l'art contemporain occidental actuel : une certaine transgression ", Essais, n 7, Laetitia Biscarrat et Clément Dussarps (éd.), « Normes communiquées, normes communicantes ", 2015, p. 94. 
La subversion et l'audace qu'autorise la culture d'élite sont-elles les conditions suffisantes pour un retournement des normes hégémoniques? Déconstruisant la dichotomie naïve entre une culture populaire standardisée et une culture des élites émancipée, Pascale Camaerbeke montre que le discours et l'esthétique de la subversion n'affranchissent pas des normes. Créée pour le Festival d'Avignon, la pièce de théâtre de Jan Lauwers La chambre d'Isabella est une exubérante ode à la vie qui a fait l'objet d'un succès retentissant du public et de la critique. Elle met en scène des transgressions, notamment l'inceste entre une femme âgée de 89 ans et son petit-fils. L'auteure montre que la pièce, malgré cette subversion ultime, contribue paradoxalement à la réactivation d'une norme raciste, héritière des imaginaires coloniaux occidentaux, qu'incarne le personnage de Vendredi, un gigolo noir dont le pénis fascine l'héroïne blanche ${ }^{22}$. Son analyse révèle que les normes sont mises en jeu dans la pièce selon des modalités ironiques et citationnelles qui, à l'instar des normes de genre évoquées précédemment, participent du maintien de normes racialisées hégémoniques.

Qu'il s'agisse du théâtre, de l'art corporel, des blogs ou des émissions de télévision, les études de ce dossier se livrent à un véritable travail critique des normes. Elles mettent au jour les mécanismes d'imposition et de négociation des normes de genre, d'ethnoracialisation, de corporéité qui sont à l'œuvre dans les productions culturelles. L’omniprésence de ces normes communicationnelles ne signifie toutefois pas leur stricte imposition sur les publics. Loin des visions mécanistes et linéaires de la communication, rappelons que les acteurs jouent leur propre partition, qui differe parfois du sens commun imposé. En d'autres termes, s'il n'y a pas d'au-delà des rapports de pouvoir, si le sujet, par sa sujétion inaugurale, et toujours déjà assujetti à des normes, celles-ci font l'objet d'usages au cours desquels elles sont parfois bousculées, discutées, re-signifiées. Conditionnées à leur itérabilité, les normes sont un pouvoir régulateur qui porte en lui les possibles de sa propre transformation. Une question essentielle, tournée vers les acteurs, se pose alors : "qu'est-ce-que les gens en font? "

Laetitia Biscarrat

EA 4426 MICA

Université Jean Monnet de Saint-Étienne laetitiabiscarrat@hotmail.com

Clément Dussarps

EA 4426 MICA

Université Bordeaux Montaigne clement.dussarps@gmail.com

22 Sur la société plantocratique du XVIII siècle comme laboratoire des identités modernes voir Elsa Dorlin, La matrice de la race. Généalogie sexuelle et coloniale de la nation française, Paris, La Découverte, 2006. 